\title{
Legless Locomotion: Models and Experimental Demonstration
}

\author{
Ravi Balasubramanian, Alfred A. Rizzi, and Matthew T. Mason \\ \{bravi, arizzi, matt.mason\}@cs.cmu.edu \\ Robotics Institute, Carnegie Mellon University, \\ Pittsburgh, PA 15213, USA.
}

\begin{abstract}
We show through experiment and simulation that a high-centered round-bodied legged robot can locomote by generating out-of-phase motions of reaction masses attached to its legs. These leg motions create body attitude oscillations which, when coupled with the slipfree contact constraints, locomote the robot. By varying the mean position of the leg oscillations, the robot can move in different directions in the plane. We also present some simplified models, where body attitude dynamics and contact kinematics are decoupled, to explain this form of legless locomotion.
\end{abstract}

Keywords: Legless locomotion, Lagrangian dynamics, contact kinematics, underactuated system.

\section{INTRODUCTION}

Legged robots offer good mobility, but they can get stuck. For example, when the robot body is highcentered and its legs dangle in air, the robot cannot use its legs to locomote in a conventional manner. Is a high-centered robot stuck permanently? Can the robot locomote while high-centered? In Balasubramanian et al. [1], we hypothesized that a high-centered roundbodied legged robot could locomote using oscillating reaction masses to excite body attitude oscillations. We then explored the relationship between leg motions and body attitude dynamics in simplified models using simulation. In this paper, we show both through simulation and experiment that a high-centered round-bodied legged robot can effectively locomote using these ideas. We also present models to explore the underlying mechanics in this form of locomotion.

The high-centered robot used in this paper is RRRobot (Rocking and Rolling Robot), a hemispherical shell with two short actuated legs (see Fig. 1). The massless legs have reaction masses at their distal ends similar to halteres, the dumbbells sometimes used by athletes to give impetus in leaping. The battery and processor are attached to the hemisphere bottom, and the goal is to locomote RRRobot in the plane. Fig. 2 shows a sequence of interleaved $\mathrm{X}$ and $\mathrm{Z}$ body rotations that produces predominantly forward translation. Similarly, a

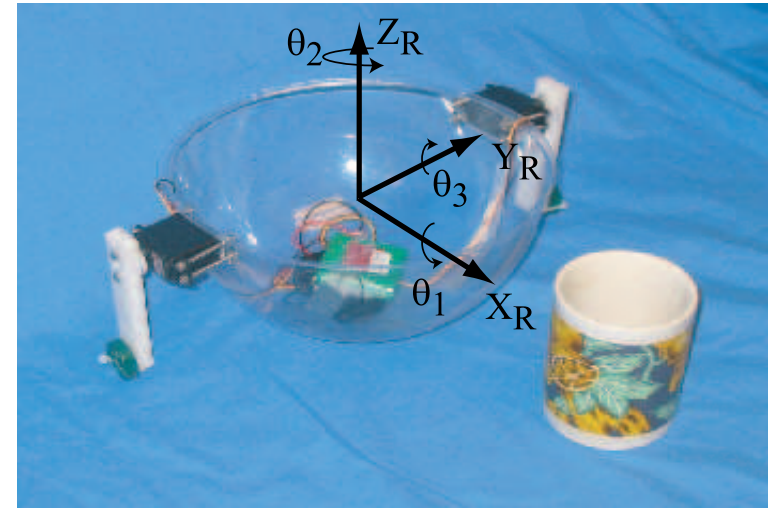

Fig. 1. The RRRobot is a hemisphere with two short actuated legs [1].

sequence of interleaved $\mathrm{Y}$ and $\mathrm{Z}$ body rotations produces predominantly sideways translation. The challenge is to find leg trajectories that create body attitude oscillations which, when coupled with the nonholonomic contact constraints, cause RRRobot to locomote in the plane. Note that producing $\mathrm{Z}$ rotations is a crucial common element of both $\mathrm{XZ}$ and $\mathrm{YZ}$ body oscillations that produce RRRobot locomotion.

An important element in RRRobot locomotion is the non-constant system inertia which changes with leg configuration (even in the body frame). Note that while gravity acts as an external force for $\mathrm{X}$ and $\mathrm{Y}$ rotations, no external forces exist to create $\mathrm{Z}$ rotations. Thus, the only way to produce $\mathrm{Z}$ rotations is by moving the legs in an out-of-phase manner and exploiting inertia differences (see [1] for more details).

After a brief review of related work, we explore the RRRobot model in Section 2 and a simplified model called the Pivoting Dynamics Model in Section 3. The Pivoting Dynamics model decouples RRRobot dynamics from the contact kinematics and can be used to understand how leg motion influences body attitude dynamics. Section 4 presents three leg trajectories that produce RRRobot locomotion with results from simulation and experiment. 


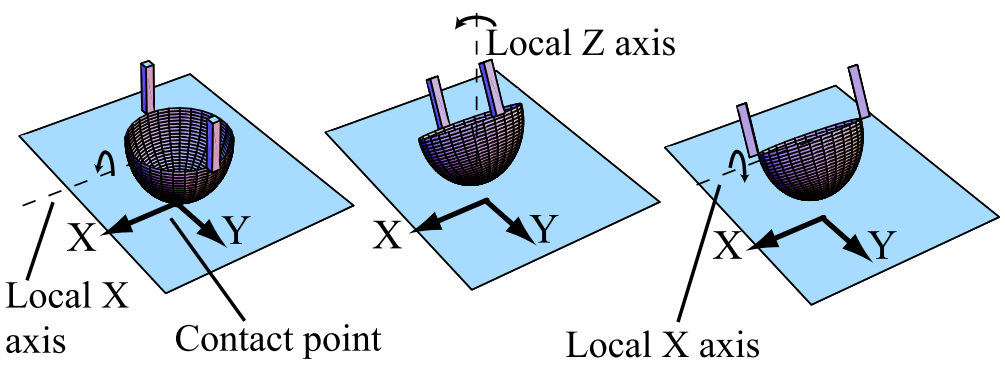

at start

(a)

(b)

(c)

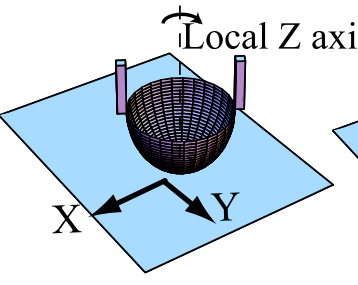

Contact point Contact point at start

(d) at end

(e)

Fig. 2. Body XZ rotations that produce locomotion. Motions are represented as rotations about axes attached to the body but aligned with the world coordinate frame. There is a local X rotation between positions (a) and (b) and positions (c) and (d); there is a local $\mathrm{Z}$ rotation between positions (b) and (c) and positions (d) and (e).

\section{A. Related Work}

The locomotion strategy proposed here for RRRobot involves the interplay between XYZ attitude dynamics and the kinematic nonholonomic contact constraints. Numerous investigators have studied dynamic systems with constraints using Lagrangian dynamics [6] or the energy-momentum method. Lewis et al. [8] study the constrained mechanics of the constant inertia snakeboard, a modified version of a skateboard in which wheel directions can be controlled. The snakeboard rider locomotes by twisting his body back and forth, while simultaneously moving the wheels with a suitable phase relationship. Lewis et al. present numerical simulations of snakeboard locomotion using characteristic wheel motions and discuss a general framework for studying mechanical systems with constraints in a coordinate-free form. Zenkov et al. [14] discuss the energy-momentum method for control of dynamic systems with nonholonomic constraints such as the rattleback, the roller racer, and the rolling disk. After identifying system symmetries, Zenkov et al. use the momentum equation to analyze the system. In studying RRRobot dynamics, we use the Lagrangian method.

While RRRobot's spatial position and orientation (called the fiber space [3]) are not actuated, the internal configuration of the two legs (called the base space) is actuated. Ostrowski [12] presents a general framework for studying systems where the fiber space can be represented as a group. Since only the base space is actuated, Ostrowski finds a connection relating the base space velocities to the fiber velocities. While Ostrowski focusses on systems with constant inertias and simple constraints, such as the snakeboard and the Hirose snake, RRRobot has a spherical contact with the plane, its inertias change with configuration, and the mass matrix is coupled. Thus, it is unclear if this framework can be extended to encompass RRRobot behavior.
RRRobot locomotes by rolling its round body without slip on the planar surface. The curvatures of the two surfaces and the type of contact between the two surfaces determine the kinematic constraints and, hence, the relative motion between the two bodies. Montana [10] derives equations for the motion of the contact point between two moving rigid bodies using differential geometry. Camicia et al. [5] provide an analysis of the nonholonomic kinematics and dynamics of the Sphericle [2], a hollow ball driven on a planar surface by an unicycle placed inside. The Sphericle and RRRobot have similar nonholonomic contact constraints (see [11] and [9] for more details on nonholonomic constraints).

If we consider RRRobot to be floating in space and ignore the surface it is resting on, the problem reduces to controlling body attitude using halteres. Fernandes et al. [7] discuss near-optimal nonholonomic motion planning for coupled bodies using Lagrangian dynamics. Given an arbitrary starting point, Fernandes et al. find plans to land a falling cat on its feet, subject to the angular momentum conservation nonholonomic constraint. RRRobot's body attitude is not directly actuated, but its inertias change with leg position. By repeatedly wiggling the legs while exploiting the differences in angular inertia, RRRobot may be able to adjust its orientation. In contrast, when spinning reaction wheels are used to control satellite attitude, the inertias of the satellite system do not change with rotation of reaction wheels [13].

To understand the interactions between leg motions and body attitude changes, we simplify the RRRobot-ona-plane model by decoupling the body attitude dynamics from the contact kinematics (see Section II-B for more details). This approach of splitting up the dynamics from the kinematics is different from the kinematic reduction technique of Bullo et al. [4] for mechanical systems with constraints. Kinematic reduction is useful because 


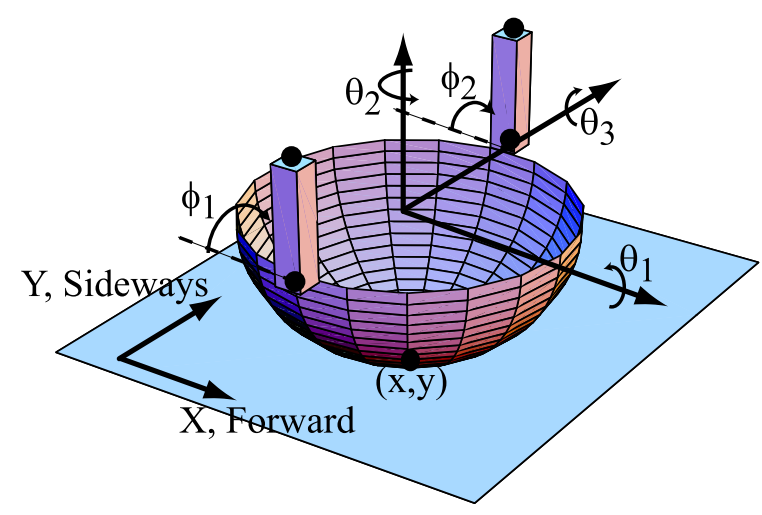

Fig. 3. RRRobot on a plane.

the kinematic reduced system is easier to control using velocity inputs than the unreduced dynamic system using acceleration inputs (see [4] for details on controllability properties for reducible systems).

\section{LEGLESS LOCOMOTION MODELS}

\section{A. RRRobot on a plane}

We begin studying legless locomotion by exploring RRRobot on a plane. The RRRobot-on-a-plane model is a hemispherical shell with two short actuated legs (see Fig. 3). The massless shell has radius $r$, and the massless legs have length $l$. There are five masses on the robot indicated by black dots: a mass at the distal end of each leg $\left(M_{l}\right)$, a mass where each leg is pinned $\left(M_{s}\right)$, and a mass at the bottom of the shell $\left(M_{b}\right)$. Torques $\tau_{1}$ and $\tau_{2}$ may be applied at the leg joints, and the shell rolls on the plane without slip.

The configuration of RRRobot on a plane $q$ consists of the sphere's position and orientation $(x, y, R)$ with respect to a spatial frame and the internal configuration of its legs $\left(\phi_{1}, \phi_{2}\right)$. Here $R=R\left(\theta_{1}, \theta_{2}, \theta_{3}\right) \in$ $S O(3)$ represents the orientation of the sphere according to the ZXY fixed-angle convention [6]. Thus, $q=$ $\left(x, y, R\left(\theta_{1}, \theta_{2}, \theta_{3}\right), \phi_{1}, \phi_{2}\right)^{T} \in \mathbb{R}^{2} \times S O(3) \times \mathbb{R}^{2}$.

The equations of motion for RRRobot on a plane take the form

$$
\begin{array}{r}
M(q) \ddot{q}+C(q, \dot{q}) \dot{q}+G(q)= \\
\tau+\left(\lambda_{1} \omega^{1}\right)^{T}+\left(\lambda_{2} \omega^{2}\right)^{T},
\end{array}
$$

where $M(q) \in \mathbb{R}^{7 \times 7}$ is the positive-definite non-diagonal variable mass matrix, $C(q, \dot{q}) \dot{q} \in \mathbb{R}^{7}$ is the vector of Coriolis and centrifugal terms, $G(q) \in \mathbb{R}^{7}$ is the vector of gravitational terms, $\tau=\left(0,0,0,0,0, \tau_{1}, \tau_{2}\right)^{T}$ is the generalized force, and $\omega^{a} \in \mathbb{R}^{7}, a=1,2$, are the rolling constraints for a sphere-plane contact [10] given by

$$
\begin{aligned}
\omega^{1} & =\left(1,0,0, r \sin \theta_{1},-r, 0,0\right), \\
\omega^{2} & =\left(0,1, r \cos \theta_{3}, r \cos \theta_{1} \sin \theta_{3}, 0,0,0\right),
\end{aligned}
$$

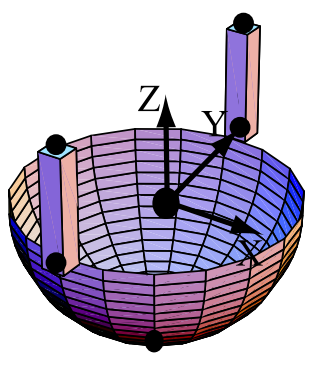

(a)

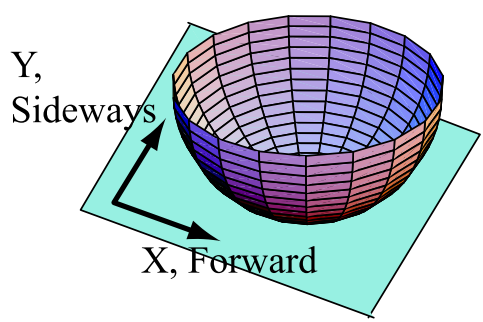

(b)
Fig. 4. The Pivoting Dynamics model simplifi es the RRRobot-ona-plane model (see Figure 3) into two parts: (a) RRRobot pivoted at its geometric center on a spherical joint and (b) a sphere on a plane.

and $\lambda_{a} \in \mathbb{R}$ is the magnitude of the contact constraint force. The right side of (1) indicates that only the legs are actuated.

\section{B. Pivoting Dynamics Model}

The RRRobot-on-a-plane model includes the interplay between body dynamics and contact kinematics. To analyze just the interaction between leg motion and body attitude, we simplify the RRRobot-on-a-plane model by pivoting the robot on a spherical joint and ignoring the effect of translation on body attitude dynamics. Once we compute the body attitude motion for a certain leg trajectory, we use the contact kinematics equations to approximately predict RRRobot translation in the plane. Thus, this model, called the Pivoting Dynamics model, approximately reduces the RRRobot system into two parts (see Fig. 4): 1) The dynamics of RRRobot rotating about a spherical joint, 2) The contact kinematics of a sphere on the plane.

The configuration of the Pivoting Dynamics model $q_{p}$ consists of the sphere's orientation $R\left(\theta_{1}, \theta_{2}, \theta_{3}\right)$ with respect to a spatial frame and the configuration of its legs $\left(\phi_{1}, \phi_{2}\right)$. Thus, $q_{p}=\left(R\left(\theta_{1}, \theta_{2}, \theta_{3}\right), \phi_{1}, \phi_{2}\right)^{T} \in$ $S O(3) \times \mathbb{R}^{2}$.

The equations of motion for the Pivoting Dynamics model take the form

$$
M\left(q_{p}\right) \ddot{q}_{p}+C\left(q_{p}, \dot{q}_{p}\right) \dot{q}_{p}+G\left(q_{p}\right)=\tau
$$

where $M\left(q_{p}\right) \in \mathbb{R}^{5 \times 5}$ is the positive-definite nondiagonal variable mass matrix, $C\left(q_{p}, \dot{q}_{p}\right) \in \mathbb{R}^{5}$ is the vector of Coriolis and centrifugal terms, $G\left(q_{p}\right) \in \mathbb{R}^{5}$ is the vector of gravitational terms, and $\tau=\left(0,0,0, \tau_{1}, \tau_{2}\right)^{T}$ is the generalized force. The right side of (3) indicates that only the legs are actuated and that there are no constraints on the system. Once we compute the changes in body configuration for a certain leg trajectory, we use 


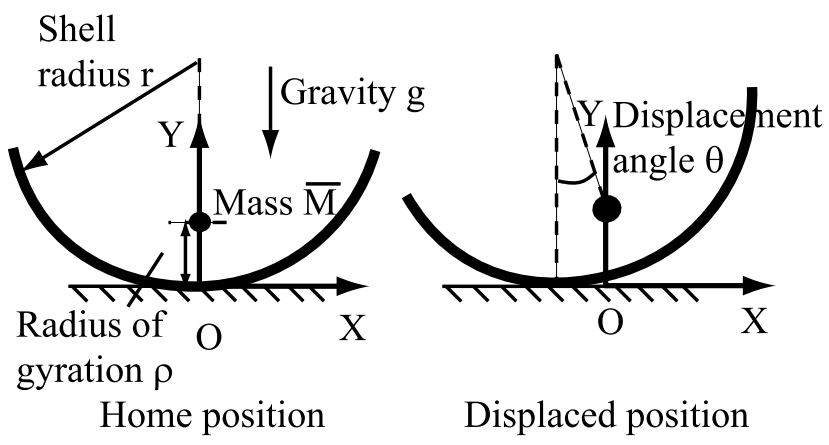

Fig. 5. A planar eccentric-mass wheel performs harmonic oscillations for small amplitude.

the kinematic contact equations

$$
\left(\begin{array}{c}
\omega^{1} \\
\omega^{2}
\end{array}\right) \dot{q}=\left(\begin{array}{l}
0 \\
0
\end{array}\right)
$$

to compute the velocity of the contact point in the plane, where $\omega^{a}$ is given in (2).

\section{Single Axis Rotation Models}

If we consider body attitude changes only about one axis, say, the $\mathrm{X}$ or $\mathrm{Y}$ axis, then RRRobot on a plane is similar to a planar wheel with an eccentric mass (see Fig. 5). The location of the mass and the inertia of the system is determined by the weight distribution on the robot. If $r$ is the wheel radius, $\bar{M}$ is the lumped mass of the system, and $\rho$ is the radius of gyration of the system with respect to an axis passing through the contact point and perpendicular to the plane, the time-period for small amplitudes is

$$
T_{w}=2 \pi \sqrt{\frac{\rho^{2}}{g(r-\rho)}},
$$

where $g$ is gravity. Note that $T_{w}$ decreases as $r$ increases, and $T_{w}$ increases as $\rho$ increases.

If the Pivoting Dynamics model is restricted to oscillate about the $\mathrm{X}$ or $\mathrm{Y}$ axis, then the Pivoting Dynamics model is similar to a simple pendulum (see Fig. 6), whose time-period is

$$
T_{s p}=2 \pi \sqrt{\frac{\rho}{g}},
$$

where $\rho$ is the radius of gyration, and $g$ is gravity. The time-period $T_{s p}$ decreases as $\rho$ decreases.

Note that to get similar oscillatory behavior between the eccentric mass wheel and the simple pendulum, a rearrangement of masses may be required. Table I shows the time-periods for $\mathrm{X}$ and $\mathrm{Y}$ rotations for the RRRoboton-a-plane model and the Pivoting Dynamics model. Since the time periods of the two models are close to each other, we do not rearrange the masses.

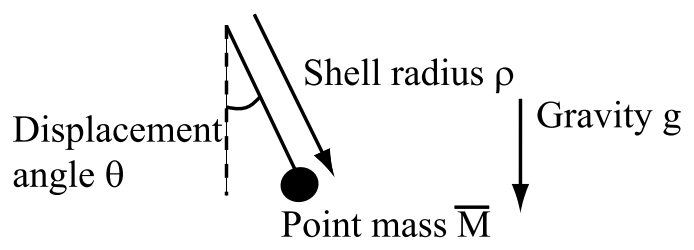

Fig. 6. The simple pendulum performs harmonic oscillations for small amplitude.

TABLE I

ROTATION TIME-PERIODS FOR THE RRROBOT-ON-A-PLANE MOdEL AND THE PIVOTING DYNAMICS MODEL

\begin{tabular}{|c|c|c|}
\hline & X Rotations $(\mathrm{sec})$ & Y Rotations $(\mathrm{sec})$ \\
\hline RRRobot-on-a-plane & 1.29 & 1.07 \\
Pivoting Dynamics & 1.19 & 0.96 \\
\hline
\end{tabular}

\section{PRODUCING RRRobot TRANSLATION}

There are two oscillators in the RRRobot system due to gravity- the $\mathrm{X}$ attitude oscillator and the $\mathrm{Y}$ attitude oscillator. It seems natural to use sinusoidal leg trajectories or gaits to control the body oscillatory motion. We present three sinusoidal gaits of the form $a \sin (\omega t+\beta)+\gamma$ that produce RRRobot translation. In all three gaits, the legs are $\pi / 2$ out-of-phase with each other. The legs oscillate about the vertical position in Gait 1, the horizontal position in Gait 2, and $\pi / 4$ off the horizontal in Gait 3 (see Table II for the gait parameters). We ran simulations of the RRRobot-on-a-plane model and the Pivoting Dynamics model for fifty seconds, while we ran the robot experiment for one hundred seconds. The robot starts from rest at the origin with the legs in the vertical position, and a PD controller is used to track the position trajectories. We use $M_{s}=0.053 \mathrm{~kg}, M_{l}=0.057 \mathrm{~kg}$, $M_{b}=0.3 \mathrm{~kg}, r=0.12 \mathrm{~m}, l=0.1 \mathrm{~m}, g=9.81 \mathrm{~m} / \mathrm{s}$, and a damping coefficient $k=-0.01$. In the experiments, a vertical tether provides the servo power and control signals. We keep body oscillations small to minimize external disturbances from the tether. In simulation and experiment, we observe initial transients in the robot motion.

\section{Gait 1}

Gait 1 produces translation along the $\mathrm{Y}$ axis (see Fig. 7) due to $Y Z$ body attitude oscillations, while $X$ body attitude changes are negligible.

\section{Gait 2}

Gait 2 produces translation along the $\mathrm{X}$ axis (see Fig. 8) due to XZ body attitude oscillations, while $\mathrm{Y}$ body attitude changes are negligible. There is a large $\mathrm{X}$ translation at the start, because the legs move quickly from the vertical position to sinusoidal oscillations about 
TABLE II

GAIT PARAMETERS

\begin{tabular}{|c|c|c|}
\multicolumn{3}{c}{ Gait 1} \\
\hline & Leg 1 & Leg 2 \\
\hline Amplitude $a(\mathrm{rad})$ & 0.3 & 0.3 \\
Frequency $\omega(\mathrm{rad} / \mathrm{s})$ & 8 & 8 \\
Phase $\beta(\mathrm{rad})$ & 0 & $\pi / 2$ \\
Offset angle $\gamma(\mathrm{rad})$ & $\pi / 2$ & $\pi / 2$ \\
\hline
\end{tabular}

\begin{tabular}{|c|c|c|} 
Gait 2 \\
\hline & Leg 1 & Leg 2 \\
\hline Amplitude $a(\mathrm{rad})$ & 0.3 & 0.3 \\
Frequency $\omega(\mathrm{rad} / \mathrm{s})$ & 8 & 8 \\
Phase $\beta(\mathrm{rad})$ & 0 & $\pi / 2$ \\
Offset angle $\gamma(\mathrm{rad})$ & 0 & 0 \\
\hline
\end{tabular}

\begin{tabular}{|c|c|c|} 
Gait 3 \\
\hline & Leg 1 & Leg 2 \\
\hline Amplitude $a(\mathrm{rad})$ & 0.3 & 0.3 \\
Frequency $\omega(\mathrm{rad} / \mathrm{s})$ & 8 & 8 \\
Phase $\beta(\mathrm{rad})$ & 0 & $\pi / 2$ \\
Offset angle $\gamma(\mathrm{rad})$ & $\pi / 4$ & $\pi / 4$ \\
\hline
\end{tabular}

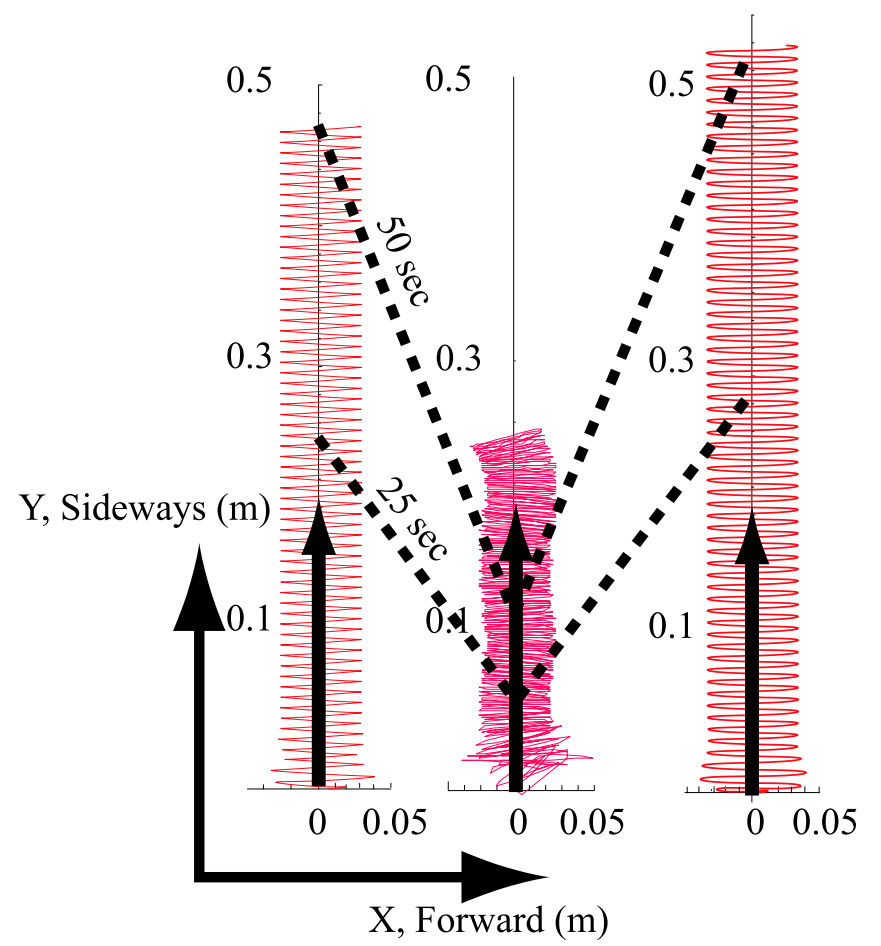

(a)

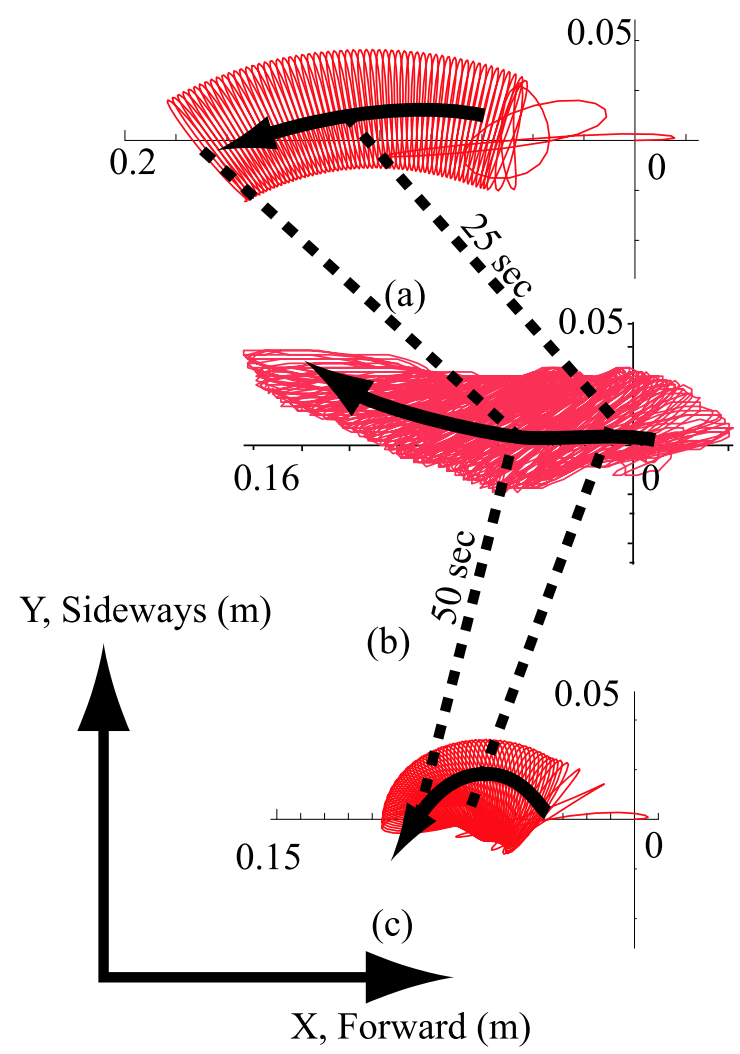

Fig. 8. Planar plots of contact point time history during forwards locomotion produced by Gait 2 in (a) RRRobot-on-a-plane simulation, (b) RRRobot-on-a-plane experiment, (c) Pivoting Dynamics model simulation. The solid arrow gives robot motion direction, and the dotted lines indicate the robot position at the specifi ed time.

the horizontal. Gait 2 does not produce much translation, because the $\mathrm{XZ}$ oscillations are small and surface stickiness restricts motion. Our experience indicates that this gait is the least reliable of the gaits explored in this paper.

Gait 3

Gait 3 produces counter-clockwise circular translation (see Fig. 9) due to a combination of XYZ body attitude oscillations. The robot completes a circle in the RRRobot-on-a-plane simulation, completes one and a half circles in the Pivoting Dynamics Model simulation, and almost completes a half circle in experiment.

Note that in all three gaits, swapping the relative phase between the two legs produces translation in the opposite direction. The paths followed by the contact point in simulation and experiment match well, but there is one clear difference- the robot in experiment moves slower than in simulation. This may be due to unmodelled surface friction, slip between the body and the surface, or a deformed spherical shape at the contact point.

The translation produced in the Pivoting Dynamics model and in the RRRobot-on-a-plane model match
Fig. 7. Planar plots of contact point time history during sideways locomotion produced by Gait 1 in (a) RRRobot-on-a-plane simulation, (b) RRRobot-on-a-plane experiment, (c) Pivoting Dynamics model simulation. The solid arrow gives robot motion direction, and the dotted lines indicate the robot position at the specifi ed time. 


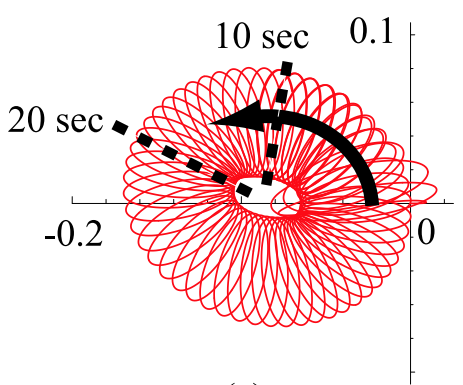

(a)
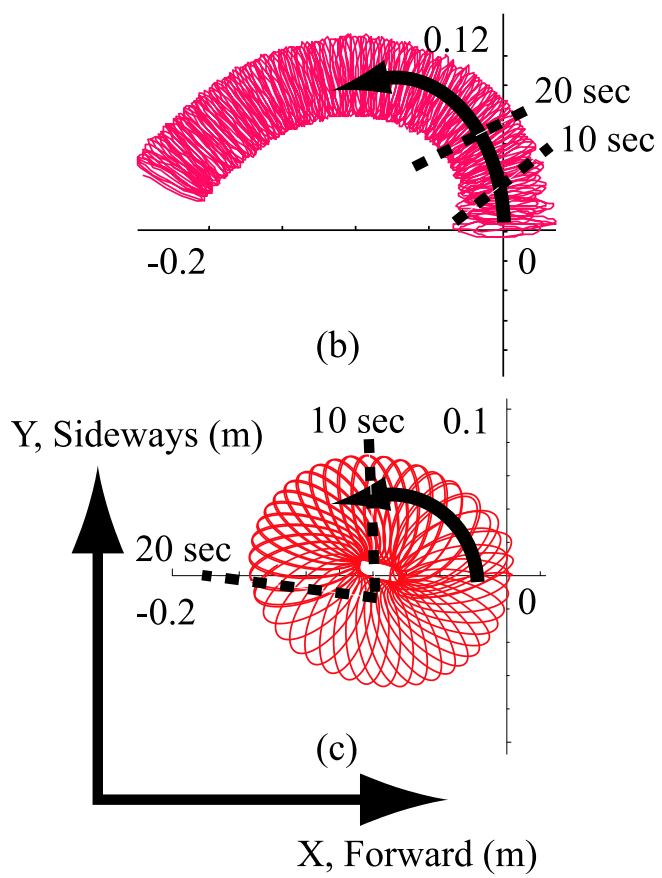

Fig. 9. Planar plots of contact point time history during forwards locomotion produced by Gait 3 in (a) RRRobot-on-a-plane simulation, (b) RRRobot-on-a-plane experiment, (c) Pivoting Dynamics model simulation. The solid arrow gives robot motion direction, and the dotted lines indicate the robot position at the specifi ed time.

well; the contact point follows similar paths, but the Pivoting Dynamics model moves faster, especially for Gaits 1 and 3. This is because the Pivoting Dynamics Model is pivoted at its geometric center, while in the RRRobot-on-a-plane Model, the robot has a rolling contact. Thus, for a given change in attitude, the point of contact moves faster in the Pivoting Dynamics model than in the RRRobot-in-a-plane Model. In summary, we can use the Pivoting Dynamics model to approximate RRRobot planar translation.

\section{Conclusion}

We explored locomotion for a high-centered roundbodied legged robot, the RRRobot, using experiments and simulation. We presented sinusoidal leg trajectories that produce forward, sideways, and rotational translation and explored simplified models to understand the locomotion. Future work will include using more legs to perform richer motion, understanding the influence of body shape on the translation, gait search, and kinematic reduction of RRRobot dynamics.

\section{ACKNOWLEDGMENT}

This work was supported under NSF IIS 0082339, NSF IIS 0222875, and DARPA/ONR N00014-98-1-0747 contracts. Devin Balkcom gave insightful comments on the dynamics and nonholonomy of mechanical systems, Brendan Meeder helped build the robot and tracking code, and Siddhartha Srinivasa gave useful comments on the paper.

\section{REFERENCES}

[1] R. Balasubramanian, A. A. Rizzi, and M. T. Mason. Legless locomotion for legged robots. In Proceedings of the International Conference on Robots and Intelligent Systems, volume 1, pages 880-885, 2003.

[2] A. Bicchi, A. Balluchi, D. Prattichizzo, and A. Gorelli. Introducing the sphericle: an experimental testbed for research and teaching in nonholonomy. In Proceedings of the IEEE International Conference on Robotics and Automation, pages 2620-2625, 1997.

[3] A. Bloch, J. Baillieul, P.Crouch, and J. Marsden. Nonholonomic Mechanics and Control. Springer, 2003.

[4] F. Bullo, A. D. Lewis, and K. M. Lynch. Controllable kinematic reductions for mechanical systems: concepts, computational tools, and examples. In Mathematical Theory of Networks and Systems, aug 2002.

[5] C. Camicia, F. Conticelli, and A. Bicchi. Nonholonomic kinematics and dynamics of the sphericle. In Proceedings of the IEEE International Conference on Intelligent Robots and Systems, pages 805-810, 2000.

[6] J. J. Craig. Introduction to Robotics. Addison Wesley, 1989.

[7] C. Ferandes, L. Gurvits, and Z. Li. Near optimal nonholonomic motion planning for a system of coupled rigid bodies. IEEE Transactions on Automatic Control, 39(3), 1994.

[8] A. Lewis, J. Ostrowski, R. Murray, and J. Burdick. Nonholonomic mechanics and locomotion: The snakeboard example. In Proceedings of the International Conference on Robotics and Automation, volume 3, pages 2391-2397, 1994.

[9] Z. Li and J. Canny. Motion of two rigid bodies with rolling constraint. IEEE Transactions on Robotics and Automation, 6(1):62-72, Feb. 1990.

[10] D. J. Montana. The kinematics of contact and grasp. The International Journal of Robotics Research, 7(3):17-32, June 1988.

[11] R. M. Murray, Z. X. Li, and S. S. Sastry. A Mathematical Introduction to Robotic Manipulation. CRC Press, 1994.

[12] J. P. Ostrowski. The Mechanics and Control of Undulatory Robotic Locomotion. PhD thesis, California Institute of Technology, 1996.

[13] C. Rui, I. V. Kolmanovsky, and N. H. McClamroch. Nonlinear attitude and shape control of spacecraft with articulated appendages and reaction wheels. IEEE Transactions on Automatic Control, 45(8):1455-69, Aug. 2000.

[14] D. Zenkov, A. Bloch, and J. Marsden. The energy-momentum method for the stability of nonholonomic systems. Technical report, California Institute of Technology, 1997. 